\title{
LIGHT-FRONT HOLOGRAPHY AND THE LIGHT-FRONT SCHRÖDINGER EQUATION*
}

\author{
STANLEY J. BRODSKY \\ SLAC National Accelerator Laboratory, \\ Stanford University \\ Stanford, CA 94309, USA \\ sjbth@slac.stanford.edu \\ GUY DE TÉRAMOND \\ Universidad de Costa Rica \\ San José, Costa Rica \\ gdt@asterix.crnet.cr
}

\begin{abstract}
One of the most important nonperturbative methods for solving QCD is quantization at fixed light-front time $\tau=t+z / c$ - Dirac's "Front Form". The eigenvalues of the light-front QCD Hamiltonian predict the hadron spectrum and the eigensolutions provide the light-front wavefunctions which describe hadron structure. More generally, we show that the valence Fock-state wavefunctions of the light-front QCD Hamiltonian satisfy a single-variable relativistic equation of motion, analogous to the nonrelativistic radial Schrödinger equation, with an effective confining potential $U$ which systematically incorporates the effects of higher quark and gluon Fock states. We outline a method for computing the required potential from first principles in QCD. The holographic mapping of gravity in AdS space to QCD, quantized at fixed light-front time, yields the same light front Schrödinger equation; in fact, the soft-wall AdS/QCD approach provides a model for the light-front potential which is color-confining and reproduces well the light-hadron spectrum. One also derives via light-front holography a precise relation between the bound-state amplitudes in the fifth dimension of AdS space and the boost-invariant light-front wavefunctions describing the internal structure of hadrons in physical space-time. The elastic and transition form factors of the pion and the nucleons are found to be well described in this framework. The light-front AdS/QCD holographic approach thus gives a frame-independent first approximation of the color-confining dynamics, spectroscopy, and excitation spectra of relativistic light-quark bound states in QCD.
\end{abstract}

\section{INTRODUCTION: LIGHT-FRONT QCD}

A central goal of hadron and nuclear physics is to compute the spectrum and dynamics of hadrons from first principles in QCD. One of the most important nonperturbative methods for solving QCD is quantization at fixed light-front time $\tau=x^{+}=t+z / c$ - Dirac's "Front Form". [1] Solving a quantum field theory is equivalent to determining the eigensolutions of the light-front (LF) Hamiltonian - the relativistic field theoretic analog of the Heisenberg equation. Remarkably, one can use "Light-front holography" to show that the AdS/QCD theory based on the dynamics of a gravitational theory in the fifth dimension of anti de-Sitter (AdS) space has a direct mapping to QCD defined at fixed light-front time. [2] In fact, the light-front AdS/QCD holographic approach provides a frameindependent first approximation to the color-confining dynamics, spectroscopy, and excitation spectra of relativistic light-quark bound states in QCD.

As emphasized by Dirac, the quantization of a relativistic quantum field theory at fixed light-front time has the extraordinary advantage that boost transformations are kinematical rather than dynamical. [1] Hadronic eigenstates $P_{\mu} P^{\mu}=M^{2}$ are determined by the Lorentz-invariant Hamiltonian equation for the relativistic bound-state

$$
H_{L F}|\psi(P)\rangle=M^{2}|\psi(P)\rangle,
$$

with $H_{L F} \equiv P_{\mu} P^{\mu}=P^{-} P^{+}-\mathbf{P}_{\perp}^{2}$. The LF Hamiltonian $H_{L F}$ defined at fixed $\tau$ is a Lorentz scalar. One can derive the LF Hamiltonian directly from the QCD Lagrangian using canonical methods. [3] If one chooses light-cone gauge, the quanta of the gluon field have physical polarization $S^{z}= \pm 1$ and no ghost degrees of freedom. Solving nonperturbative QCD is thus equivalent to solving the Heisenberg matrix eigenvalue problem. Angular momentum $J^{z}$ is conserved at every vertex.

The hadronic state $|\psi\rangle$ is an expansion in multiparticle Fock states $|\psi\rangle=\sum_{n} \psi_{n}|n\rangle$, where the components $\psi_{n}=$ $\langle n \mid \psi\rangle$ are a column vector of states, and the basis vectors $|n\rangle$ are the $n$-parton eigenstates of the free LF Hamiltonian:

\footnotetext{
* This research was supported by the Department of Energy contract DE-AC02-76SF00515. SLAC-PUB-15211.
} 
$|q \bar{q}\rangle,|q \bar{q} g\rangle,|q \bar{q} q \bar{q}\rangle \cdots$ etc. This Fock space expansion of the hadronic eigensolutions of the LF Hamiltonian provides the light-front wavefunctions (LFWFs) $\psi_{n / H}\left(x_{i}, \vec{k}_{\perp i}, \lambda_{i}\right)$ which define the hadron's structure in the same sense that the Schrödinger wavefunction in nonrelativistic quantum mechanics describes the physics of atoms. Remarkably, the LFWFs are independent of the hadron's total four momentum $P^{\mu}$ and thus of the observer's Lorentz frame; no boosts are required. The Fock state expansion begins with the dominant valence state (e.g., $n=3$ for the nucleon); higher Fock state have extra gluons and $q \bar{q}$ pairs. The constituents satisfy $x_{i}=k_{i}^{+} / P^{+}, \sum_{i}^{n} x_{i}=1, \sum_{i}^{n} \vec{k}_{\perp i}=0$. The $\lambda_{i}$ specify their spin projections in the $\hat{z}$ direction, as in non-relativistic physics. Each Fock state satisfies $J^{z}$ conservation.

Hadronic observables can be computed directly from the LFWFs: structure functions and transverse momentum distributions (TMDs) are computed from the absolute squares of the LFWFs; in fact, LFWFs are the basis of the parton model. Elastic and transition form factors, decay amplitudes, and generalized parton distributions are computed from overlaps of the LFWFs. A key example of the utility of the light-front formalism is the Drell-Yan West formula $[4,5]$ for the space-like form factors of electromagnetic currents given as overlaps of initial and final LFWFs. The valence and sea quark and gluon distributions which are measured in deep inelastic lepton scattering are defined from the squares of the LFWFs summed over all Fock states $n$. The gauge-invariant "distribution amplitudes" $\phi_{H}\left(x_{i}, Q\right)$ defined from the integral over the transverse momenta $\mathbf{k}_{\perp i}^{2} \leq Q^{2}$ of the valence (smallest $n$ ) Fock state provide a fundamental measure of the hadron at the amplitude level; $[6,7]$ they are the nonperturbative inputs to the factorized form of hard exclusive amplitudes and exclusive heavy hadron decays in perturbative QCD.

At high momentum where one can iterate the hard scattering kernel, one can derive dimensional counting rules $[8,9]$ and factorization theorems. The resulting structure functions distributions obey DGLAP evolution equations, and the distribution amplitudes obey ERBL evolution equations as a function of the maximal invariant mass, thus providing a physical factorization scheme. [10] In each case, the derived quantities satisfy the appropriate operator product expansions, sum rules, and evolution equations. At large $x$ where the struck quark is far-off shell, DGLAP evolution is quenched, [11] so that the fall-off of the DIS cross sections in $Q^{2}$ satisfies Bloom-Gilman inclusive-exclusive duality at fixed $W^{2}$.

Given the light-front wavefunctions $\psi_{n / H}$, one can compute a large range of other hadron observables. Exclusive weak transition amplitudes [12] such as $B \rightarrow \ell \nu \pi$, and the generalized parton distributions [13] measured in deeply virtual Compton scattering $\gamma^{*} p \rightarrow \gamma p$ are (assuming the "handbag" approximation) overlap of the initial and final LFWFs with $n=n^{\prime}$ and $n=n^{\prime}+2$. It is also possible to compute jet hadronization at the amplitude level from first principles from the LFWFs. [14] A similar method has been used to predict the production of antihydrogen from the off-shell coalescence of relativistic antiprotons and positrons. [15]

The simplicity of the front form contrasts with the usual instant-form formalism. Current matrix elements defined at ordinary time $t$ must include the coupling of the photons and vector bosons fields to connected vacuum currents; otherwise the result is not Lorentz-invariant. Thus knowledge of the hadronic eigensolutions of the instant-form Hamiltonian are insufficient for determining form factors or other observables. In addition, the boost of an instant form wavefunction from $p$ to $p+q$ changes particle number and is an extraordinarily complicated dynamical problem.

In some cases such as the T-odd Sivers single-spin asymmetry in semi-inclusive deep inelastic scattering [16], DrellYan lepton-pair production [17, 18], and single-particle inclusive reactions, one must include the "lensing" (Wilson-line) effects of initial or final-state interactions [19]. The ordinary rules of factorization are broken since the sign of the single spin asymmetry depends on whether one has initial-state or final-state lensing $[17,18]$. Diffractive deep inelastic scattering at leading twist is also due to the color-neutralization of final state lensing. Double-initial state interactions break the Lam-Tung relation at leading twist [20].

The LFWFs of hadrons thus provide a direct connection between observables and the QCD Lagrangian. They are as essential to hadron physics as DNA is to biology!

Unlike lattice gauge theory, the LF Hamiltonian formalism is frame-independent, has no fermion-doubling, and is performed in Minkowski space. The LF vacuum is defined as the state of lowest invariant mass and is trivial up to zero modes. There are no quark or gluon vacuum condensates - the corresponding physics is contained within the LFWFs themselves. [21, 22]

Theories such as $Q C D(1+1)$ can be solved to any desired accuracy for any number of colors, flavors, and quark masses by diagonalization of the Heisenberg LF matrix by employing periodic boundary conditions as in the discretized light-cone quantization method (DLCQ) [23, 24]. Solving QCD $(3+1)$ using DLCQ is much more challenging since the matrix diagonalization yields all hadronic eigensolutions simultaneously. Vary et al. [25] are developing an alternative method which uses a basis of orthonormal harmonic states generated by AdS/QCD. A LF coupled cluster method for solving quantum field theories has been developed by Chabysheva and Hiller. [26] We will discuss in the next section a new method where one can solve for each hadron eigenstate in terms of a single-variable LF equation analogous to the radial Schrödinger equation in atomic physics. We shall also show that, remarkably, one obtains a single-variable equation of the same form with a color-confining potential using AdS/QCD and LF Holography. [2] In all of these LF methods, the observer's frame is arbitrary. 
Other advantages of light-front quantization include:

- The simple structure of the light-front vacuum allows an unambiguous definition of the partonic content of a hadron in QCD. The chiral and gluonic condensates can be identified with the hadronic LF Fock states, [21, 27] rather than vacuum properties. LF zero mode contributions are also possibly relevant. [28] As shown by a Bethe Salpeter analysis [29], the usual Gell-Mann-Oakes-Renner (GMOR) relation [30] is satisfied in QCD, but with the vacuum to vacuum matrix element $\langle 0|q \bar{q}| 0\rangle$ replaced by $\left\langle 0\left|q \gamma_{5}\right| \pi\right\rangle / f_{\pi}$. This matrix element requires a pion $q \bar{q}$ LF wavefunction with $L^{z}=1, S^{z}=-1$ or $L^{z}=-1, S^{z}=+1$, which occurs when a quark mass insertion causes a quark chiral flip. For a recent review see Ref. [31].

- Since it is defined along the front of a light-wave, at fixed $\tau$, the LF front-form vacuum is causal, and it is thus a match to the empty universe with zero cosmological constant. In contrast, the instant-form vacuum describes a state of indeterminate energy at fixed time $t$, a boundary condition outside the causal horizon. The QCD LF vacuum is trivial up to zero modes in the front form, thus eliminating contributions to the cosmological constant. [21] In the case of the Higgs model, the effect of the usual Higgs vacuum expectation value is replaced by a constant $k^{+}=0$ zero mode field. [32]

- If one quantizes QCD in the physical light-cone gauge (LCG) $A^{+}=0$, then the gluons have physical angular momentum projections $S^{z}= \pm 1$. The orbital angular momenta of quarks and gluons are defined unambiguously, and there are no ghosts.

- The gauge-invariant distribution amplitude $\phi(x, Q)$ is the integral of the valence LFWF in LCG integrated over the internal transverse momentum $k_{\perp}^{2}<Q^{2}$ because the Wilson line is trivial in this gauge. It is also possible to quantize QCD in Feynman gauge in the light front. [33]

- LF Hamiltonian perturbation theory provides a simple method for deriving analytic forms for the analog of Parke-Taylor amplitudes [34] where each particle spin $S^{z}$ is quantized in the LF $z$ direction. The gluonic $g^{6}$ amplitude $T(-1-1 \rightarrow+1+1+1+1+1+1)$ requires $\Delta L^{z}=8$; it thus must vanish at tree level since each three-gluon vertex has $\Delta L^{z}= \pm 1$. However, the order $g^{8}$ one-loop amplitude can be nonzero.

- Amplitudes in light-front perturbation theory may be automatically renormalized using the "alternate denominator" subtraction method. [35] The application to QED has been checked at one and two loops. [35]

- A fundamental theorem for gravity can be derived from the equivalence principle: the anomalous gravitomagnetic moment defined from the spin-flip matrix element of the energy-momentum tensor is identically zero, $B(0)=$ 0. [36] This theorem can be proven in the light-front formalism Fock state by Fock state. [37]

- LFWFs obey the cluster decomposition theorem, providing an elegant proof of this theorem for relativistic bound states. [38]

- LF quantization provides a distinction [39] between static (the square of LFWFs) distributions versus nonuniversal dynamic structure functions, such as the Sivers single-spin correlation and diffractive deep inelastic scattering which involve final state interactions. The origin of nuclear shadowing and process-independent anti-shadowing also becomes explicit. [40]

- LF quantization provides a simple method to implement jet hadronization at the amplitude level. [14]

- The instantaneous fermion interaction in LF quantization provides a simple derivation of the $J=0$ fixed pole contribution to deeply virtual Compton scattering, [41] i.e., the $e_{q}^{2} s^{0} F(t)$ contribution to the DVCS amplitude which is independent of photon energy and virtuality.

- Unlike instant time quantization, the bound state Hamiltonian equation of motion in the LF is frame independent. This makes a direct connection of QCD with AdS/CFT methods possible. [2]

\section{THE LIGHT-FRONT SCHRÖDINGER EQUATION: A SEMICLASSICAL APPROXIMATION TO QCD}

It is greatly advantageous to reduce the full multiparticle eigenvalue problem of the LF Hamiltonian to an effective light-front Schrödinger equation (LFSE) which acts on the valence sector LF wavefunction and determines each eigensolution separately. [42] In contrast, diagonalizing the LF Hamiltonian yields all eigensolutions simultaneously, a complex task. The central problem for deriving the LFSE becomes the derivation of the effective interaction $U$ 
which acts only on the valence sector of the theory and has, by definition, the same eigenvalue spectrum as the initial Hamiltonian problem. For carrying out this program one most systematically express the higher Fock components as functionals of the lower ones. The method has the advantage that the Fock space is not truncated and the symmetries of the Lagrangian are preserved. [42]

For example, we can derive the LFSE for a meson in terms of the invariant impact-space variable for a two-parton state $\zeta^{2}=x(1-x) \mathbf{b}_{\perp}^{2}$

$$
\psi(x, \zeta, \varphi)=e^{i L \varphi} X(x) \frac{\phi(\zeta)}{\sqrt{2 \pi \zeta}},
$$

thus factoring the angular dependence $\varphi$ and the longitudinal, $X(x)$, and transverse mode $\phi(\zeta)$. In the limit of zero quark masses the longitudinal mode decouples and the LF eigenvalue equation $P_{\mu} P^{\mu}|\phi\rangle=M^{2}|\phi\rangle$ is thus a light-front wave equation for $\phi$

$$
\left[-\frac{d^{2}}{d \zeta^{2}}-\frac{1-4 L^{2}}{4 \zeta^{2}}+U\left(\zeta^{2}, J, M^{2}\right)\right] \phi_{J, L, n}\left(\zeta^{2}\right)=M^{2} \phi_{J, L, n}\left(\zeta^{2}\right),
$$

a relativistic single-variable LF Schrödinger equation.

The potential in the LFSE is determined from the two-particle irreducible (2PI) $q \bar{q} \rightarrow q \bar{q}$ Greens' function. In particular, the higher Fock states in intermediate states leads to an effective interaction $U\left(\zeta^{2}, J, M^{2}\right)$ for the valence $|q \bar{q}\rangle$ Fock state. [42] The potential $U$ thus depends on the hadronic eigenvalue $M^{2}$ via the LF energy denominators $P_{\text {initial }}^{-}-P_{\text {intermediate }}^{-}+i \epsilon$ of the intermediate states which connect different LF Fock states. Here $P_{\text {initial }}^{-}=\left(M^{2}+\mathbf{P}_{\perp}^{2}\right) / P^{+}$. The dependence of $U$ on $M^{2}$ is analogous to the retardation effect in QED interactions, such as the hyperfine splitting in muonium, which involves the exchange of a propagating photon. Accordingly, the eigenvalues $M^{2}$ must be determined self-consistently. [43] The $M^{2}$ dependence of the effective potential thus reflects the contributions from higher Fock states in the LFSE (3), since $U\left(\zeta^{2}, J, M^{2}\right)$ is also the kernel for the scattering amplitude $q \bar{q} \rightarrow q \bar{q}$ at $s=M^{2}$. It has only "proper" contributions; i.e., it has no $q \bar{q}$ intermediate state. The potential can be constructed, in principle systematically, using LF time-ordered perturbation theory. In fact, as we shall see below, the QCD theory has the identical form as the AdS theory, but with the quantum field-theoretic corrections due to the higher Fock states giving a general form for the potential. This provides a novel way to solve nonperturbative QCD. The LFSE for QCD becomes increasingly accurate as one includes contributions from very high particle number Fock states.

The above discussion assumes massless quarks. More generally we must include mass terms [44, 45] $m_{a}^{2} / x+$ $m_{b}^{2} /(1-x)$ in the kinetic energy term and allow the potential $U\left(\zeta^{2}, x, J, M^{2}\right)$ to have dependence on the LF momentum fraction $x$. The quark masses also appear in $U$ due to the presence in the LF denominators as well as the chiralityviolating interactions connecting the valence Fock state to the higher Fock states. In this case, however, the equation of motion cannot be reduced to a single variable.

The LFSE approach also can be applied to atomic bound states in QED and nuclei. In principle one could compute the spectrum and dynamics of atoms, such as the Lamb shift and hyperfine splitting of hydrogenic atoms to high precision by a systematic treatment of the potential. Unlike the ordinary instant form, the resulting LFWFs are independent of the total momentum and can thus describe "flying atoms" without the need for dynamical boosts, such as the "true muonium" $\left(\mu^{+} \mu^{-}\right)$bound states which can be produced by Bethe-Heitler pair production $\gamma \rightarrow\left(\mu^{+} \mu^{-}\right) Z$ below threshold. [46] A related approach for determining the valence light-front wavefunction and studying the effects of higher Fock states without truncation has been given in Ref. [26].

\section{EFFECTIVE CONFINEMENT INTERACTION FROM THE GAUGE/GRAVITY CORRESPONDENCE}

A remarkable correspondence between the equations of motion in AdS and the Hamiltonian equation for relativistic bound-states was found in Ref. [2]. In fact, to a first semiclassical approximation, LF QCD is formally equivalent to the equations of motion on a fixed gravitational background [2] asymptotic to $\mathrm{AdS}_{5}$, where confinement properties are encoded in a dilaton profile $\varphi(z)$.

A spin- $J$ field in $\operatorname{AdS}_{d+1}$ is represented by a rank $J$ tensor field $\Phi_{M_{1} \cdots M_{J}}$, which is totally symmetric in all its 
indices. In presence of a dilaton background field $\varphi(z)$ the action is ${ }^{1}$

$$
\begin{aligned}
S=\frac{1}{2} \int d^{d} x d z \sqrt{g} e^{\varphi(z)}\left(g^{N N^{\prime}} g^{M_{1} M_{1}^{\prime}} \cdots g^{M_{J} M_{J}^{\prime}} D_{N} \Phi_{M_{1} \cdots M_{J}} D_{N^{\prime}} \Phi_{M_{1}^{\prime} \cdots M_{J}^{\prime}}\right. & \\
& \left.-\mu^{2} g^{M_{1} M_{1}^{\prime}} \cdots g^{M_{J} M_{J}^{\prime}} \Phi_{M_{1} \cdots M_{J}} \Phi_{M_{1}^{\prime} \cdots M_{J}^{\prime}}+\cdots\right),
\end{aligned}
$$

where $M, N=1, \cdots, d+1, \sqrt{g}=(R / z)^{d+1}$ and $D_{M}$ is the covariant derivative which includes parallel transport. The coordinates of AdS are the Minkowski coordinates $x^{\mu}$ and the holographic variable $z$ labeled $x^{M}=\left(x^{\mu}, z\right)$. The $\mathrm{d}+$ 1 dimensional mass $\mu$ is not a physical observable and is a priory an arbitrary parameter. The dilaton background field $\varphi(z)$ in (4) introduces an energy scale in the five-dimensional AdS action, thus breaking its conformal invariance. It vanishes in the conformal ultraviolet limit $z \rightarrow 0$.

A physical hadron has plane-wave solutions and polarization indices along the $3+1$ physical coordinates $\Phi_{P}(x, z)_{\mu_{1} \cdots \mu_{J}}=e^{-i P \cdot x} \Phi(z)_{\mu_{1} \cdots \mu_{J}}$, with four-momentum $P_{\mu}$ and invariant hadronic mass $P_{\mu} P^{\mu}=M^{2}$. All other components vanish identically. One can then construct an effective action in terms of the spin modes $\Phi_{J}=\Phi_{\mu_{1} \mu_{2} \cdots \mu_{J}}$ with only physical degrees of freedom. In this case the system of coupled differential equations which follow from (4) reduce to a homogeneous equation in terms of the physical field $\Phi_{J}$ upon rescaling the AdS mass $\mu$

$$
\left[-\frac{z^{d-1-2 J}}{e^{\varphi(z)}} \partial_{z}\left(\frac{e^{\varphi}(z)}{z^{d-1-2 J}} \partial_{z}\right)+\left(\frac{\mu R}{z}\right)^{2}\right] \Phi(z)_{J}=M^{2} \Phi(z)_{J} .
$$

Upon the substitution $z \rightarrow \zeta$ and $\phi_{J}(\zeta)=(\zeta / R)^{-3 / 2+J} e^{\varphi(z) / 2} \Phi_{J}(\zeta)$ in (5), we find for $d=4$ the LFSE (3) with effective potential [49]

$$
U\left(\zeta^{2}, J\right)=\frac{1}{2} \varphi^{\prime \prime}\left(\zeta^{2}\right)+\frac{1}{4} \varphi^{\prime}\left(\zeta^{2}\right)^{2}+\frac{2 J-3}{2 \zeta} \varphi^{\prime}\left(\zeta^{2}\right),
$$

provided that the fifth dimensional mass $\mu$ is related to the internal orbital angular momentum $L=\max \left|L^{z}\right|$ and the total angular momentum $J^{z}=L^{z}+S^{z}$ according to $(\mu R)^{2}=-(2-J)^{2}+L^{2}$. The critical value $L=0$ corresponds to the lowest possible stable solution, the ground state of the LF Hamiltonian. For $J=0$ the five dimensional mass $\mu$ is related to the orbital momentum of the hadronic bound state by $(\mu R)^{2}=-4+L^{2}$ and thus $(\mu R)^{2} \geq-4$. The quantum mechanical stability condition $L^{2} \geq 0$ is thus equivalent to the Breitenlohner-Freedman stability bound in AdS. [50] The scaling (twist) dimensions are $2+L$ independent of $J$, in agreement with the twist-scaling dimension of a two-parton bound state in QCD.

The correspondence between the LF and AdS equations thus determines the effective confining interaction $U$ in terms of the infrared behavior of AdS space and gives the holographic variable $z$ a kinematical interpretation. The identification of the orbital angular momentum is also a key element of our description of the internal structure of hadrons using holographic principles.
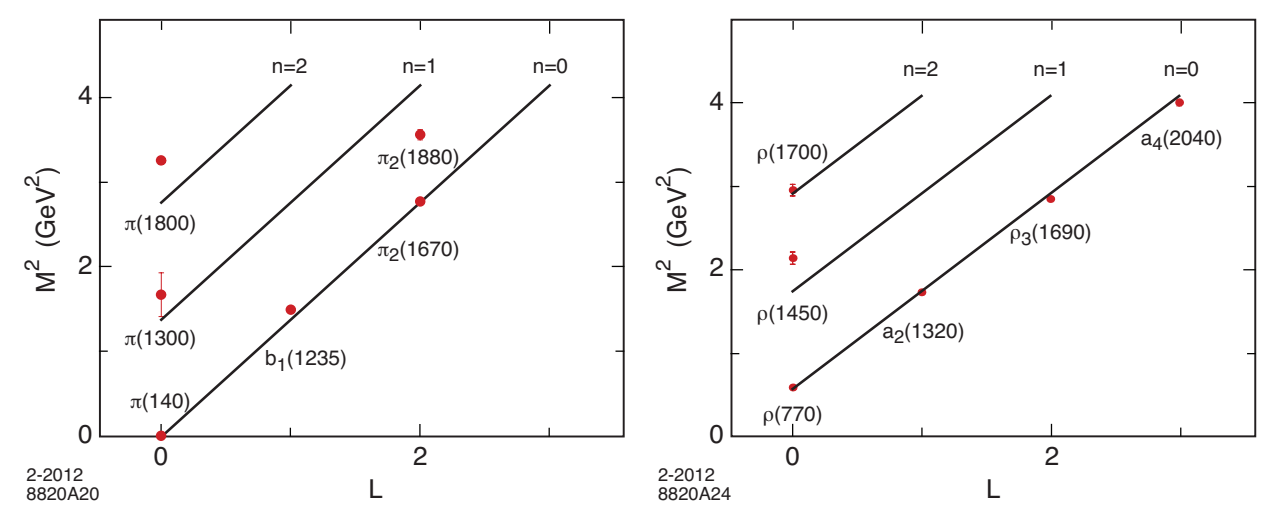

FIG. 1: $I=1$ parent and daughter Regge trajectories for the $\pi$-meson family (left) with $\kappa=0.59 \mathrm{GeV}$; and the $\rho$-meson family (right) with $\kappa=0.54 \mathrm{GeV}$.

\footnotetext{
1 The study of higher integer and half-integer spin wave equations in AdS is based on our collaboration with Hans Guenter Dosch. [47] See also the discussion in Ref. [48].
} 
A particularly interesting example is a dilaton profile $\exp \left( \pm \kappa^{2} z^{2}\right)$ of either sign, since it leads to linear Regge trajectories [51] and avoids the ambiguities in the choice of boundary conditions at the infrared wall. For the confining solution $\varphi=\exp \left(\kappa^{2} z^{2}\right)$ the effective potential is $U\left(\zeta^{2}, J\right)=\kappa^{4} \zeta^{2}+2 \kappa^{2}(J-1)$ and Eq. (3) has eigenvalues $M_{n, J, L}^{2}=$ $4 \kappa^{2}\left(n+\frac{J+L}{2}\right)$, with a string Regge form $M^{2} \sim n+L$. A discussion of the light meson and baryon spectrum, as well as the elastic and transition form factors of the light hadrons using LF holographic methods, is given in Ref. [52]. As an example the spectral predictions for the $J=L+S$ light pseudoscalar and vector meson states are compared with experimental data in Fig. 1 for the positive sign dilaton model. The data is from PDG. [53] The results for $Q^{4} F_{1}^{p}\left(Q^{2}\right)$ and $Q^{4} F_{1}^{n}\left(Q^{2}\right)$ are shown in Fig. 2. We also show in Fig. 2 the results for $F_{2}^{p}\left(Q^{2}\right)$ and $F_{2}^{n}\left(Q^{2}\right)$ for the same value of $\kappa$ normalized to the static quantities $\chi_{p}$ and $\chi_{n}$. To compare with physical data we have shifted the poles appearing in the expression of the form factor to their physical values located at $M^{2}=4 \kappa^{2}(n+1 / 2)$ following the discussion in Ref. [52]. The value $\kappa=0.545 \mathrm{GeV}$ is determined from the $\rho$ mass. The data compilation is from Ref. [54].
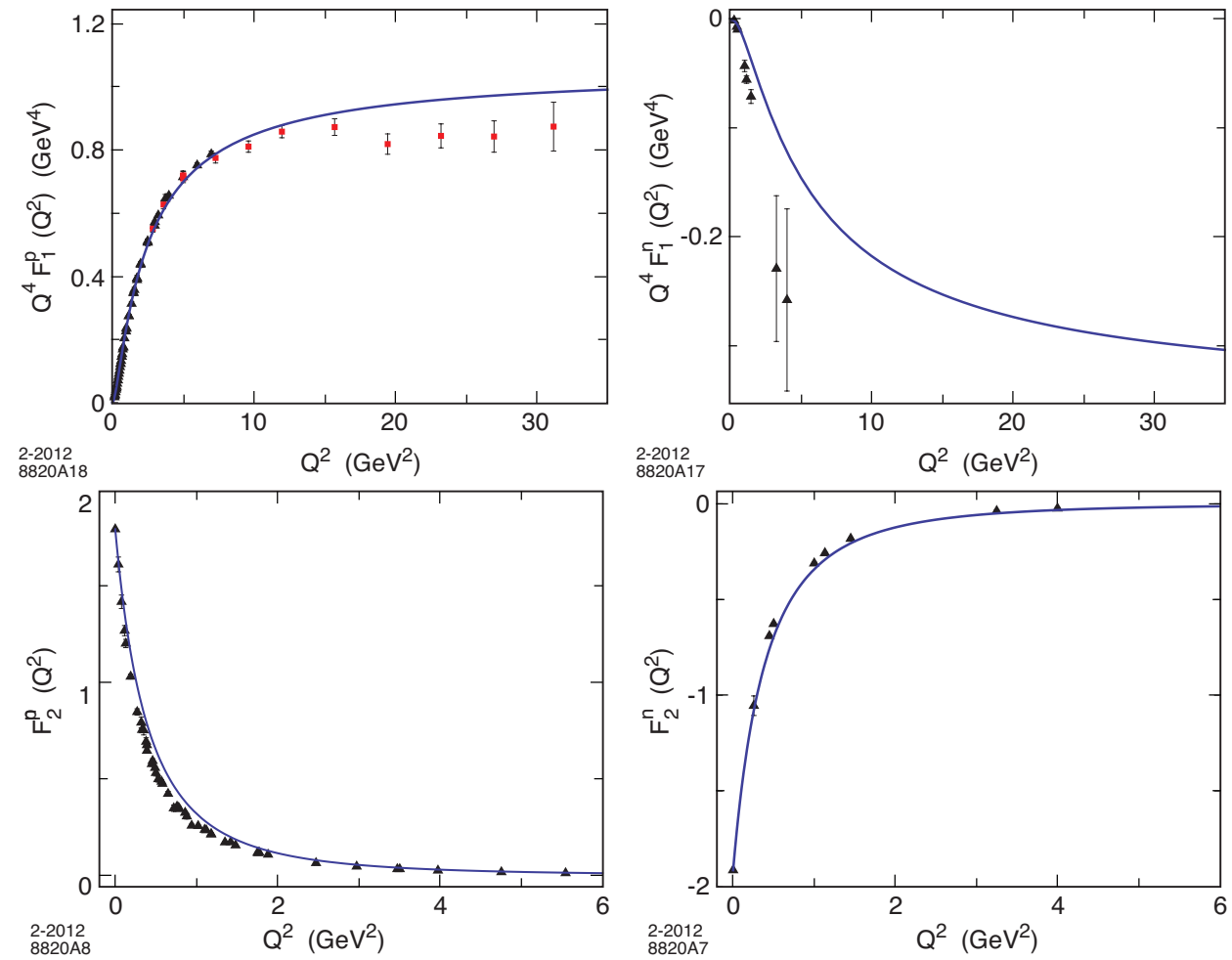

FIG. 2: Predictions for $Q^{4} F_{1}^{p}\left(Q^{2}\right)$ (upper left) and $Q^{4} F_{1}^{n}\left(Q^{2}\right)$ (upper right) in the soft wall model. The results for $F_{2}^{p}\left(Q^{2}\right)$ (lower left) and $F_{2}^{n}\left(Q^{2}\right)$ (lower right) are normalized to static quantities. The nucleon spin-flavor structure corresponds to the $S U(6)$ limit.

Despite some limitations of AdS/QCD, the LF holographic approach to the gauge/gravity duality, has given significant physical insight into the strongly-coupled nature and internal structure of hadrons. In particular, the AdS/QCD soft-wall model provides an elegant analytic framework for describing nonperturbative hadron dynamics, the systematics of the excitation spectrum of hadrons, including their empirical multiplicities and degeneracies. It also provides powerful new analytical tools for computing hadronic transition amplitudes incorporating conformal scaling behavior at short distances and the transition from the hard-scattering perturbative domain, where quark and gluons are the relevant degrees of freedom, to the long-range confining hadronic region.

The effective interaction $U\left(\zeta^{2}\right)=\kappa^{4} \zeta^{2}+2 \kappa^{2}(J-1)$ that is derived from the AdS/QCD model is instantaneous in LF time and acts on the lowest state of the LF Hamiltonian. This equation describes the spectrum of mesons as a function of $n$, the number of nodes in $\zeta^{2}$ and the total angular momentum $J=J^{z}$, with $J^{z}=L^{z}+S^{z}$ the sum of the internal orbital angular momentum of the constituents ${ }^{2}$ and their internal spin. It is the relativistic frame-

\footnotetext{
2 The $S O(2)$ Casimir $L^{2}$ corresponds to the group of rotations in the transverse LF plane.
} 
independent front-form analog of the non-relativistic radial Schrödinger equation for muonium and other hydrogenic atoms in presence of an instantaneous Coulomb potential.

The AdS/QCD harmonic oscillator potential could in fact emerge from the exact QCD formulation when one includes contributions from the LFSE potential $U$ which are due to the exchange of two connected gluons; i.e., "H" diagrams. [55] We notice that $U$ becomes complex for an excited state since a denominator can vanish; this gives a complex eigenvalue and the decay width.

\section{FUTURE APPLICATIONS}

The AdS/QCD model can be considered as a first approximation to the full QCD dynamics of hadrons. The analytic form of the LFWFs obtained by solving the LFSE for meson and baryons can be utilized for many fundamental issues in hadron physics and can be tested experimentally for a wide range of observables. ${ }^{3}$

These include

- Hadronization at the amplitude level [14]

- Resolving the factorization uncertainty in DGLAP evolution and fragmentation

- Behavior of the QCD running coupling at soft scales [57]

- The computation of transition form factors such as photon-to-meson form factors [58] and heavy-hadron weak decays

- The physics of higher Fock states including intrinsic heavy quark excitations [59]

- Hidden-color degrees of freedom in nuclear wavefunctions [60]

- Color transparency phenomena $[61,62]$

- The sublimation of gluon degrees of freedom at low virtuality [63]

- The shape of valence structure functions, distribution amplitudes, and generalized parton distributions [64]

- The emergence of dimensional counting rules at $x \rightarrow 1$ in inclusive reactions and hard exclusive reactions [65]

- New insights into the breaking of chiral symmetry [66]

- Renormalization group properties under conformal transformations [67]

\section{SUMMARY}

We have shown that the valence Fock-state wavefunctions of the eigensolutions of the light-front QCD Hamiltonian satisfy a single-variable relativistic equation of motion, analogous to the nonrelativistic radial Schrödinger equation with an effective confining potential $U$, which systematically incorporates the effects of higher quark and gluon Fock states. We have outlined a method for computing the required potential from first principles. The holographic mapping of gravity in AdS space to QCD, quantized at fixed light-front time, yields the same LF Schrödinger equation; in fact, the soft-wall AdS/QCD approach provides a model for the LF potential which is color-confining and reproduces well the light-hadron spectrum. One also derives via light-front holography a precise relation between the bound-state amplitudes in the fifth dimension of AdS space and the boost-invariant light-front wavefunctions describing the internal structure of hadrons in physical space-time. The elastic and transition form factors of the pion and the nucleons are well described in this framework. The light-front AdS/QCD holographic approach thus gives a frame-independent first approximation of the color-confining dynamics, spectroscopy, and excitation spectra of relativistic light-quark bound states in QCD. This provides the basis for a profound connection between physical QCD, quantized on the light-front, and the physics of hadronic modes in a higher dimensional AdS space.

\footnotetext{
${ }^{3}$ A recent application of holographic LFWFs to diffractive rho meson electroproduction is given in Ref. [56].
} 


\section{Acknowledgments}

This research was supported by the Department of Energy contract DE-AC02-76SF00515. Invited talk, presented by SJB at the QCD Evolution Workshop, May 14 - 17, 2012, at the Thomas Jefferson National Accelerator Facility, Newport News, Virginia. SLAC-PUB-15211.

[1] P. A. M. Dirac, Rev. Mod. Phys. 21, 392 (1949).

[2] G. F. de Teramond and S. J. Brodsky, Phys. Rev. Lett. 102, 081601 (2009) [arXiv:0809.4899 [hep-ph]].

[3] S. J. Brodsky, H. C. Pauli and S. S. Pinsky, Phys. Rept. 301, 299 (1998) [arXiv:hep-ph/9705477].

[4] S. D. Drell and T. M. Yan, Phys. Rev. Lett. 24, 181 (1970).

[5] G. B. West, Phys. Rev. Lett. 24, 1206 (1970).

[6] G. P. Lepage and S. J. Brodsky, Phys. Lett. B 87, 359 (1979).

[7] A. V. Efremov and A. V. Radyushkin, Phys. Lett. B 94, 245 (1980).

[8] S. J. Brodsky and G. R. Farrar, Phys. Rev. Lett. 31, 1153 (1973).

[9] V. A. Matveev, R. M. Muradian and A. N. Tavkhelidze, Lett. Nuovo Cim. 7, 719 (1973).

[10] G. P. Lepage and S. J. Brodsky, Phys. Rev. D 22, 2157 (1980).

[11] S. J. Brodsky and G. P. Lepage, SLAC-PUB-2294 (1979).

[12] S. J. Brodsky and D. S. Hwang, Nucl. Phys. B 543, 239 (1999) [arXiv:hep-ph/9806358].

[13] S. J. Brodsky, M. Diehl and D. S. Hwang, Nucl. Phys. B 596, 99 (2001) [arXiv:hep-ph/0009254].

[14] S. J. Brodsky, G. de Teramond and R. Shrock, AIP Conf. Proc. 1056, 3 (2008) [arXiv:0807.2484 [hep-ph]].

[15] C. T. Munger, S. J. Brodsky and I. Schmidt, Phys. Rev. D 49, 3228 (1994).

[16] S. J. Brodsky, D. S. Hwang and I. Schmidt, Phys. Lett. B 530, 99 (2002) [arXiv:hep-ph/0201296].

[17] J. C. Collins, Phys. Lett. B 536, 43 (2002) [arXiv:hep-ph/0204004].

[18] S. J. Brodsky, D. S. Hwang and I. Schmidt, Nucl. Phys. B 642, 344 (2002) [arXiv:hep-ph/0206259].

[19] S. J. Brodsky, B. Pasquini, B. W. Xiao and F. Yuan, Phys. Lett. B 687, 327 (2010) [arXiv:1001.1163 [hep-ph]].

[20] D. Boer, S. J. Brodsky and D. S. Hwang, Phys. Rev. D 67, 054003 (2003) [arXiv:hep-ph/0211110].

[21] S. J. Brodsky and R. Shrock, Proc. Nat. Acad. Sci. 108, 45 (2011) [arXiv:0905.1151 [hep-th]].

[22] S. J. Brodsky, C. D. Roberts, R. Shrock, P. Tandy, Phys. Rev. C82, 022201 (2010) [arXiv:1005.4610 [nucl-th]].

[23] H. C. Pauli and S. J. Brodsky, Phys. Rev. D 32, 2001 (1985).

[24] K. Hornbostel, S. J. Brodsky and H. C. Pauli, Phys. Rev. D 41, 3814 (1990).

[25] J. P. Vary et al., Phys. Rev. C 81, 035205 (2010) [arXiv:0905.1411 [nucl-th]].

[26] S. S. Chabysheva and J. R. Hiller, Phys. Lett. B 711, 417 (2012) [arXiv:1103.0037 [hep-ph]].

[27] A. Casher and L. Susskind, Phys. Rev. D 9, 436 (1974).

[28] T. Banks and A. Casher, Nucl. Phys. B 169, 103 (1980).

[29] P. Maris and C. D. Roberts, Phys. Rev. C 56, 3369 (1997) [nucl-th/9708029].

[30] M. Gell-Mann, R. J. Oakes and B. Renner, Phys. Rev. 175, 2195 (1968).

[31] S. J. Brodsky, C. D. Roberts, R. Shrock and P. C. Tandy, arXiv:1202.2376 [nucl-th].

[32] P. P. Srivastava and S. J. Brodsky, Phys. Rev. D 66, 045019 (2002) [arXiv:hep-ph/0202141].

[33] P. P. Srivastava and S. J. Brodsky, Phys. Rev. D 61, 025013 (2000) [hep-ph/9906423].

[34] L. Motyka and A. M. Stasto, Phys. Rev. D 79, 085016 (2009) [arXiv:0901.4949 [hep-ph]].

[35] S. J. Brodsky, R. Roskies and R. Suaya, Phys. Rev. D 8, 4574 (1973).

[36] O. V. Teryaev, arXiv:hep-ph/9904376.

[37] S. J. Brodsky, D. S. Hwang, B. Q. Ma and I. Schmidt, Nucl. Phys. B 593, 311 (2001) [arXiv:hep-th/0003082].

[38] S. J. Brodsky and C. R. Ji, Phys. Rev. D 33, 2653 (1986).

[39] S. J. Brodsky, Nucl. Phys. A 827, 327C (2009) [arXiv:0901.0781 [hep-ph]].

[40] S. J. Brodsky, I. Schmidt and J. J. Yang, Phys. Rev. D 70, 116003 (2004) [arXiv:hep-ph/0409279].

[41] S. J. Brodsky, F. J. Llanes-Estrada, J. T. Londergan and A. P. Szczepaniak, arXiv:0906.5515 [hep-ph].

[42] H. C. Pauli, Eur. Phys. J. C 7, 289 (1999) [arXiv:hep-th/9809005].

[43] G. F. de Teramond and S. J. Brodsky, arXiv:1206.4365 [hep-ph].

[44] S. J. Brodsky and G. F. de Teramond, arXiv:0802.0514 [hep-ph].

[45] T. Gutsche, V. E. Lyubovitskij, I. Schmidt and A. Vega, Prog. Part. Nucl. Phys. 67, 206 (2012) [arXiv:1111.5169 [hep-ph]].

[46] S. J. Brodsky and R. F. Lebed, Phys. Rev. Lett. 102, 213401 (2009) [arXiv:0904.2225 [hep-ph]].

[47] H. G. Dosch, S .J. Brodsky and G. F. deTeramond (in preparation).

[48] T. Gutsche, V. E. Lyubovitskij, I. Schmidt, A. Vega, Phys. Rev. D 85, 076003 (2012).

[49] G. F. de Teramond and S. J. Brodsky, AIP Conf. Proc. 1296, 128 (2010) [arXiv:1006.2431 [hep-ph]].

[50] P. Breitenlohner and D. Z. Freedman, Annals Phys. 144, 249 (1982).

[51] A. Karch, E. Katz, D. T. Son and M. A. Stephanov, Phys. Rev. D 74, 015005 (2006) [arXiv:hep-ph/0602229].

[52] G. F. de Teramond and S. J. Brodsky, arXiv:1203.4025 [hep-ph].

[53] J. Beringer et al [Particle Data Group], Phys. Rev. D86, 010001 (2012). 
[54] M. Diehl, Nucl. Phys. Proc. Suppl. 161, 49 (2006) [arXiv:hep-ph/0510221].

[55] T. Appelquist, M. Dine and I. J. Muzinich, Phys. Lett. B 69, 231 (1977).

[56] J. R. Forshaw and R. Sandapen, arXiv:1203.6088 [hep-ph], to appear in Physical Review Letters.

[57] S. J. Brodsky, G. F. de Teramond and A. Deur, Phys. Rev. D 81, 096010 (2010) [arXiv:1002.3948 [hep-ph]].

[58] S. J. Brodsky, F. G. Cao and G. F. de Teramond, Phys. Rev. D 84, 075012 (2011) [arXiv:1105.3999 [hep-ph]].

[59] W. C. Chang and J. C. Peng, Phys. Lett. B 704, 197 (2011) [arXiv:1105.2381 [hep-ph]].

[60] S. J. Brodsky, C. R. Ji and G. P. Lepage, Phys. Rev. Lett. 51, 83 (1983).

[61] S. J. Brodsky and A. H. Mueller, Phys. Lett. B 206, 685 (1988).

[62] L. El Fassi et al., Phys. Lett. B 712, 326 (2012) [arXiv:1201.2735 [nucl-ex]].

[63] S. J. Brodsky and G. F. de Teramond, PoS QCD -TNT-II, 008 (2011) [arXiv:1112.4212 [hep-th]].

[64] A. Vega, I. Schmidt, T. Gutsche and V. E. Lyubovitskij, Phys. Rev. D 85, 096004 (2012) [arXiv:1202.4806 [hep-ph]].

[65] S. J. Brodsky, M. Burkardt and I. Schmidt, Nucl. Phys. B 441, 197 (1995) [arXiv:hep-ph/9401328].

66] J. Kapusta, AIP Conf. Proc. 1343 (2011) 566.

[67] S. D. Glazek, Phys. Rev. D 85, 125018 (2012) [arXiv:1204.6504 [hep-th]]. 